\title{
A singularly perturbed vector-bias malaria model incorporating bed-net control
}

\author{
Sanaa Salman ${ }^{1}$
}

${ }^{1}$ Alexandria University

April 27, 2021

\begin{abstract}
A malaria transmission disease model with host selectivity and Insecticide treated bed nets (ITNs), as an intervention for controlling the disease, is formulated. Since the vector is an insect, the vector time scale is much more expeditious than the host time scale. This leads to a singularly perturbed model with two distinctive intrinsic time scales, two-slow for the host and one-fast for the vector. The basic reproduction number R0 is calculated and the local stability analysis is performed at equilibria of the model when the perturbation parameter e $>0$. The model is analyzed when e - 0 using asymptotic expansions technique. Merging bed-net control, vector-bias, and singular perturbation have a notable effect on the model dynamics. It is shown that if over $\% 30$ of humans use ITNs, malaria disease burden can be reduced. The dynamics on the slow surface indicate that the infected vectors decays very fast when $\mathrm{e}=0.001$ according to the numerical simulations.
\end{abstract}

\section{Hosted file}

Malaria.pdf available at https://authorea.com/users/410382/articles/519759-a-singularlyperturbed-vector-bias-malaria-model-incorporating-bed-net-control 